\title{
Über die Magensaft=Proteolyse.
}

III. Mitteiling. Über das Verhalten der Peptonkörper gegenüber

Proteasen verschiedener Herkunft.

Von

Tameji Hirooka.

(借成 第次)

(Aus dem Medizinisch-Chemischen Institut der Medizinischen

Fakultät der Kaiserlichen Tohoku-Universität, Sendai.

Direktor: Prof. Dr. S. Utzino.)

In Bezug auf die enzymologische Einstellung gegenüber Caseinooder Gelatopepton hat zuerst I tzi o k a $(1937)^{1)}$ und dann Mori $(1938)^{2)}$ beobachtet, dass das Pepton, das durch die wiederholte Einwirkung der Mazeration der Magenschleimhaut dargestellt wurde, weiter durch den Kaninchen-Pankreassaft oder Trypsin-Grübler hydrolysiert werden kann, während das Trypton, unter dem man die Abbauprodukte der Peptidnatur durch den Pankreassaft oder durch das Trypsin versteht, weiter nicht durch dies Enzym oder durch Pepsin-Salzsäure angegriffen

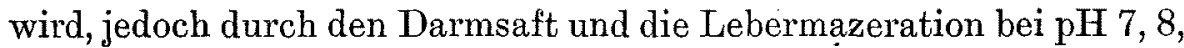
wie es auch von Chen et alt. $(1940)^{3)}$ in den Versuchen mit den durch Pankreassaft dargestelltenCaseino-undGelatotrypton bestätigt wurde.

In diesem Zusammenhang kam das Magensaft-Pepton aber sehr selten in Betracht und es wäre vom Standpunkt der Verdauungsphysiologie sehr wünschenswert, einmal das enzymologische Studium mit dem Magensaft-Pepton in Angriff zu nehmen, das man besonders dürch Einwirkung des frischen Magensaftes auf Eiweissstoffe bereitete, indem man das Verhalten des Peptons, welches durch die Mazeration der Magenschleimhaut dargestellt wurde, auch anderseits vergleichend studierte.

Dazu taucht noch eine neue Frage auf, ob das Magensaft-Pepton auch durch die Gewebeenzyme besonders durch die von der Ereptase befreite katheptische Peotonase bei schwach saurer Reaktion angreifbar ist, da es Utzin o und Sa ka i (1941) ${ }^{4}$ gelungen ist, die katheptische 
Peptonase von der ereptischen Wirkung zu trennen. Ferner wäre es auch von grossem Interesse, das Verhalten des Mucinopeptons gegenüber Proteasen an den Tag zu bringen, da Verf. in der vorigen Mitteilung über das biologische Verhalten des Mucins bzw. des Mucinopeptons auf die starke Toxität derselben hingewiesen hat.

In Hinsicht der Peptonspaltung haben Waldschmidt-Leitz und Sim on s $(1926)^{5}$ die Spaltung des Caseino-Peptons durch die Erepsinlösung und darauf Waldschmidt-Leitz und WaldschmidtGraser (1927) ${ }^{6}$ die Hydrolyse des Teleopeptons aus Fleisch durch den Hundedarmsaft festgestellt, nachdem die Peptonspaltung (Peptonum ex albumine, Merck) früher von Waldschmidt-Leitz und Mitarbeiter $\left(1925\right.$ u. 1926) ${ }^{7)}$ verneint worden war.

Neuerdings hat Matsuo (1941) ${ }^{8)}$ die Spaltung des Peptons durch den Hundedarmsaft beobachtet.

In der vorliegenden Arbeit hat Verf. sich weiter unter Leitung von Prop. S. Utzin o mit dem Studium der enzymologischen Finstellung gegenüber Magensaft-Peptonkörpern beschäftigt und die Ergebnisse hier zusammengestellt.

Wie aus der Darstellungsweise dieser Peptonkörper zu ersehen ist, die durch die maximale Spaltung des Proteins nach der wiederholten Pepsin-Salzsäure-Digestion gewonnen wurden, zeigte das Pepsin-Pepton einen starken Widerstand gegenüber der weiteren Wirkung des Magensaftes wie auch der Mazeration des Magenschleimhautbreis und es wurde in keinem Falle dadurch gespalten, während Casein im Kontrollversuch deutlich durch diesen Magensaft angegriffen wurde (Tabelle 1 u. 2).

\section{Versuchsteil.}

Tabelle 1.

Wirking des Magensaftes (Hund) und der Magenschleimhautmazeration (Kaninchen) auf Caseinopepton und Casein.

Caseinopepton: Nach maximaler Einwirkung des Magensaftes auf Casein (Hammarsten) bei pH 2,0 wurde das Digestat nach Neutralisieren mit Natronlange auf dem Wasserbade unter $40^{\circ} \mathrm{C}$ mittels Föns eingeengt dann durch Aceton gefällt und gestrocknet. Die Analysenzahlen des Caseinopeptons sind schon angegeben (H i r o o k a, 1942 I. u. II. Mitteilung).

Der Magensaft wurde aus der Magenfistel des Hundes nach der Scheinfütterung mit Fleiseh-Reis-Mahlzeit gesammelt.

Magenschleimhaut-Mazeration: 1 Teil Schleimhautbrei des Kaninchens wurde mit der 3 fachen Volummenge Glycerin-Wasser gut zerrieben, koliert und unter Toluol im Eisschrank aufbewahrt. 
Versuchsansatz mit Magensaft: $25 \mathrm{ccm} 1 \%$ iger Caseinopepton oder Caseinlösung von verschiedenen $\mathrm{pH}-$ Werten $+2 \mathrm{~cm}$ Magensaft + Toluol; digeriert bei $37^{\circ} \mathrm{C}$.

Kontrollversuch: $2 \mathrm{ccm}$ Magensaft $+25 \mathrm{ccm}$ Pufferlösung von verschiedenen pHWerten ohne Substrat + Toluol; digeriert bei $37^{\circ} \mathrm{C}$.

Versuchsansatz mit Mucosa-Mazeration: $20 \mathrm{ccm} 1 \%$ iger Caseinopepton- oder Casein-Pufferlösung von verschiedenen $\mathrm{pH}-\mathrm{Werten}+2 \mathrm{~cm}$ Magenschleimhautmazeration (Kaninchen) + Toluol; bei $37^{\circ} \mathrm{C}$ digeriert.

Kongrollversuch: $20 \mathrm{ccm}$ Pufferlösung von verschiedenen pH-Werten $+2 \mathrm{~cm}$ Magenschleimhautmazeration ohne Substrat+Toluol; digeriert bei $37^{\circ} \mathrm{C}$.

Nach einer gewissen Digestionszeit wurde die Aciditätszunabme in $4 \mathrm{ccm}$ Digestat nach Sörensen formoltitriert. Die Kontrollwerte sind von denjenigen des Hauptversuchs abgezogen. Die korrigierten Werte der Aciditätszunabme finden sich in Tabelle angegeben.

\begin{tabular}{|c|c|c|c|c|c|c|c|c|c|c|c|}
\hline \multirow[t]{2}{*}{ Substrate } & & & & Mage & saft & & & & sa-M & zerat & \\
\hline & Std. ${ }^{\mathrm{pH}}$ & 1,5 & 2 & 3 & 4 & 5 & 6 & 2 & 3 & 4 & 5 \\
\hline $\begin{array}{c}\text { Caseino- } \\
\text { pepton } \\
\text { (Saft) }\end{array}$ & $\begin{array}{r}5 \\
24 \\
72 \\
120\end{array}$ & $\begin{array}{l}0 \\
0 \\
0 \\
0,1\end{array}$ & $\begin{array}{l}0 \\
0 \\
0 \\
0\end{array}$ & $\begin{array}{l}0 \\
0 \\
0 \\
0\end{array}$ & $\begin{array}{l}0 \\
0 \\
0 \\
0\end{array}$ & $\begin{array}{l}0 \\
0 \\
0 \\
0\end{array}$ & $\begin{array}{l}0 \\
0 \\
0 \\
1\end{array}$ & $\begin{array}{l}\overline{0} \\
0 \\
0\end{array}$ & $\begin{array}{c}\overline{0} \\
0 \\
0,1\end{array}$ & $\begin{array}{l}\overline{0} \\
0 \\
0\end{array}$ & $\begin{array}{l}\overline{0} \\
0 \\
0\end{array}$ \\
\hline Casein & $\begin{array}{r}5 \\
24 \\
72 \\
120\end{array}$ & $\begin{array}{l}0,1 \\
0,2 \\
0,5 \\
0,7\end{array}$ & $\begin{array}{l}0,2 \\
0,3 \\
0,5 \\
0,5\end{array}$ & $\begin{array}{l}0,3 \\
0,4 \\
0,5 \\
0,6\end{array}$ & $\begin{array}{l}0,3 \\
0,7 \\
0,9 \\
1,0\end{array}$ & $\begin{array}{l}0,1 \\
0,1 \\
0,2 \\
0,2\end{array}$ & $\begin{array}{l}0,1 \\
0,1 \\
0,1 \\
0,1\end{array}$ & $\begin{array}{l}\overline{0,1} \\
0,3 \\
0,3\end{array}$ & $\begin{array}{l}\overrightarrow{0,3} \\
0,4 \\
0,5\end{array}$ & $\begin{array}{l}\overrightarrow{0,1} \\
0,4 \\
0,4\end{array}$ & $\begin{array}{l}\overrightarrow{0,1} \\
0,1 \\
0,1\end{array}$ \\
\hline
\end{tabular}

\section{Tabelle 2 .}

Wirküng des Magensaftes des Hundes oder der Magensehlcimhautmazeration auf Caseino-, Mucino-, Fibrino-, und Gelatopepton.

Die Peptonkörper wurden nach der maximalen Einwirkung des Hundemagensaftes oder des Magenschleimhautbreis auf Eiweisskörper dargestellt, wie es schon in der vorigen Mitteilang beschrieben war. Die Magensebleimhautmazeration besteht aus 1 Teil Schleimhautbrei des Magens und 3 Volumteilen Glycerin-Wasser $(1: 1)$.

Versuchsansatz: $20 \mathrm{~cm} 1 \%$ iger Substratpufferlösung (pH 2)+2 ccm Magensaft oder $2 \mathrm{ccm}$ Magenschleimhautmazeration + Toluol; digeriert bei $37^{\circ} \mathrm{C}$.

Kontrollversuch: $2 \mathrm{ccm}$ Magensaft oder Magenschleimhautmazeration $+20 \mathrm{ccm}$ Pufferlösung ohne Substrat+Toluol; digeriert bei $37^{\circ} \mathrm{C}$. Formoltitration.

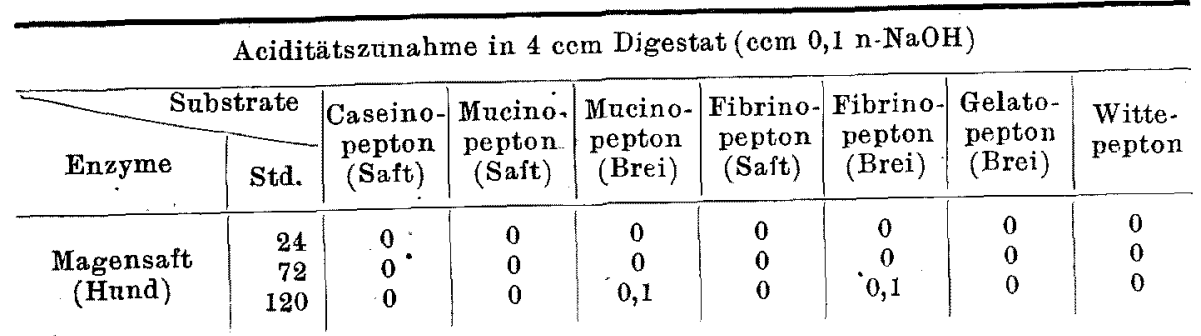




\begin{tabular}{|c|c|c|c|c|c|c|c|c|}
\hline $\begin{array}{l}\text { Magen- } \\
\text { Schleimhaut } \\
\text { (Schwein) }\end{array}$ & $\begin{array}{r}24 \\
72 \\
120\end{array}$ & $\begin{array}{l}0 \\
0 \\
0\end{array}$ & $\begin{array}{l}0 \\
0 \\
0\end{array}$ & $\begin{array}{l}0 \\
0 \\
0\end{array}$ & $\begin{array}{c}0 \\
0 \\
0,1\end{array}$ & $\begin{array}{l}0 \\
0 \\
0\end{array}$ & $\begin{array}{l}0 \\
0 \\
0\end{array}$ & $\begin{array}{l}0 \\
0 \\
0\end{array}$ \\
\hline $\begin{array}{l}\text { Magen- } \\
\text { Schleimhaut } \\
\text { (Hund) }\end{array}$ & $\begin{array}{r}24 \\
72 \\
120\end{array}$ & $\begin{array}{l}0 \\
0 \\
0\end{array}$ & E & - & 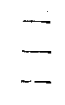 & - & - & - \\
\hline $\begin{array}{c}\text { Magen- } \\
\text { Schleimhaut } \\
\text { (Kaninchen) }\end{array}$ & $\begin{array}{r}24 \\
-72 \\
120\end{array}$ & $\begin{array}{l}0 \\
0 \\
0\end{array}$ & - & - & - & - & - & $=$ \\
\hline
\end{tabular}

Bei der Wirkung des frischen Pankreassaftes, der aus der am Hunde gelegten Pankreasfistel gewonnen und mit der von erepsinfreien Enterokinase aktiviert wurde, trat eine deutliche Hydrolyse der versuchten Peptonkörper auf, und es wurde mit Sicherheit die Spaltbarkeit des Pepsin-Peptons durch den aktivierten Pankreassaft festgestellt(Tabelle 3). Ferner ergaben die Resultate, dass die Peptonkörper durch den

$$
\text { Tabelle } 3 \text {. }
$$

Wirkung des Pankreassaftes auf Caseino-, Mucino-, Fibrino-, Gelato- und Witte-Pepton.

Die Peptonkörper wie in Tabelle 1. Der Pankreassaft wurde aus der am Hunde angelegten Pankreasfistel nach der Fleisch-Reis-Nahrung gesammelt und beim Versuche mit der erepsinfreien Enterokinase ( $\mathrm{K}$ aw a harad a) aktiviert.

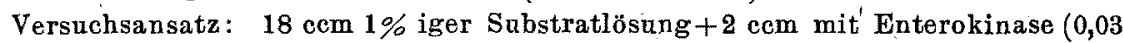
g) aktivierten Pankreassaftẹs, mit Pufferlösung auf 22 com gebracht $(\mathbf{p H} 7,8)+$ Toluol; digeriert bei $37^{\circ} \mathrm{C}$.

Kontrollversuch : $2 \mathrm{ccm}$ mit Kinase aktivierten Parkreassaftes ohne Substrat mit Puffer auf $22 \mathrm{ccm}$ gebracht ( $\mathrm{pH} \mathrm{7,8)+Toluol} \mathrm{;} \mathrm{digeriert} \mathrm{bei} 37^{\circ} \mathrm{C}$. Kontrolle mit EnzymPuffer ohne Pepton. Formoltitration. Die korrigierten Werte der Aciditutszunahme in Tabelle angegeben.

\begin{tabular}{|c|c|c|c|c|c|c|c|}
\hline \multicolumn{8}{|c|}{ Aciditätszunahme in $4 \mathrm{ccm}$ Digestat $(\mathrm{ccm} 0,1 \mathrm{n}-\mathrm{NaOH})$} \\
\hline Substrate & $\begin{array}{c}\text { Caseino- } \\
\text { pepton } \\
\text { (Saft) }\end{array}$ & $\begin{array}{l}\text { Gelato- } \\
\text { pepton } \\
\text { (Brei) }\end{array}$ & $\begin{array}{l}\text { Mucino- } \\
\text { pepton } \\
\text { (Saft) }\end{array}$ & $\begin{array}{l}\text { Mucino- } \\
\text { pepton } \\
\text { (Brei) }\end{array}$ & $\begin{array}{c}\text { Fibrino } \\
\text { pepton } \\
\text { (Saft) }\end{array}$ & $\begin{array}{l}\text { Fibrino- } \\
\text { pepton } \\
\text { (Brei) }\end{array}$ & $\begin{array}{l}\text { Witte- } \\
\text { pepton }\end{array}$ \\
\hline 24 & 0,8 & 0,5 & 0,7 & 0,3 & 0,2 & 0,7 & $0,9^{\circ}$ \\
\hline 72 & 0,9 & 0,7 & 0,8 & 0,3 & 0,3 & 0,8 & 0,9 \\
\hline 120 & 0,9 & 0,7 & 0,8 & 0,4 & 0,3 & 0,8 & 1,0 \\
\hline
\end{tabular}

frischen Darmsaft des Hundes ausserordentlich angegriffen wurden (Tabelle 4). Hier sẹi noch betont, dass die Acïditätszunahme bei der Darmsaftdigestion grösser als die bei der Pankreassaftdigestion war. 


\section{Tabelle 4.}

Wirkung des Hunde-Darmsaftes auf Caseino, Mucino-, Fibrino- und Gelatopepton.

Der Darmsaft warde aus der Thiry-Vella'schen Schlinge des Hundes nach der Reis-Fleisch-Nahrung gesammelt.

Versuchsansatz: $20 \mathrm{ccm} 1 \%$ iger Substratpufferlösung $+2 \mathrm{ccm}$ Darmsaft+Toluol ; digeriert bei $37^{\circ} \mathrm{C}$. Kontrolle mit Enzym-Puffer ohne Pepton. Formoltitration.

Die korrigierten wurde der Aciditätszunahme in Tabelle angegeben.

An dieser Stelle sei mir gestattet, Herren Prof. Dr. Satake und a. o. Prof. Dr. Wad a im physiologischen Institut für die Fistelanlegung den verbindlichsten Dank auszusprechen.

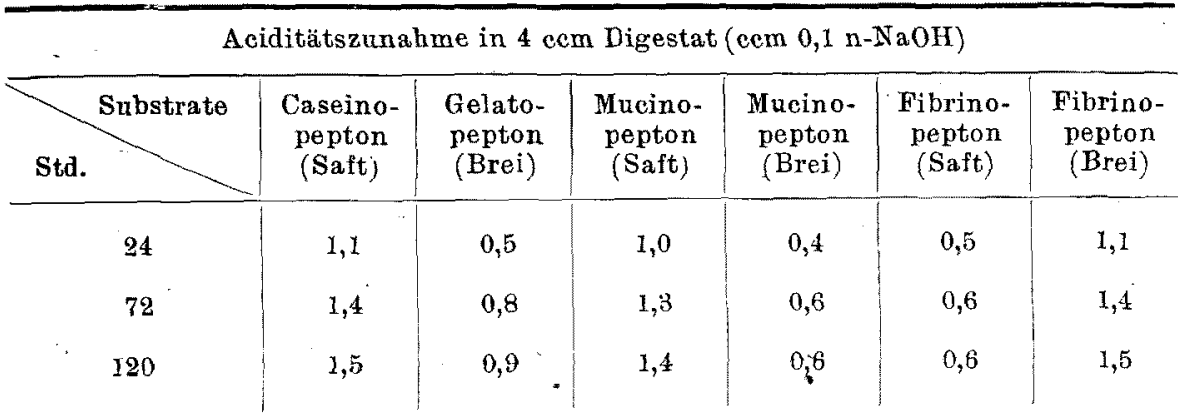

Diese Beobachtung lässt an die Angabe von Itzi ok a (1937) ${ }^{1)}$ und auch von Mori $(1938)^{2)}$ erinnern, dass das Trypton, das nach wiederholter Einwirkung des Pankreassaftes auf Eiweisskörper durch Aceton ausgefällt wurde und jeder weiteren Pankreassaftwirkung widerstand, jedoch noch weiter durch den Darmsaft angegriffen werden konnte. Man möchte deshalb eine andere und wohl weitere Einstellung des Darmsaftes als die des Pankreassaftes annehmen.

Es taucht nun eine neue Frage auf, ob die Gewebepeptidase besonders die katheptische Peptonase (Kathopeptonase) das MagensaftPepton im engeren Sinne anzugreifen imstande ist, da die Unterscheidung der Kathopeptonase von Kathepsin Utzino und Sakai (1941) im hiesigen Institut mit Erfolg gelungen ist und die Spatlung des WittePeotons durch dieses Enzym festgestellt wurde, nachdem Waldschmidt-Leitz et al. (1929) durch Kathepsin der Leber und der Milz (aktiviert oder nicht aktiviert) beobachtet hatten. Hier bediente Verf. sich zuerst der Leberereptase, die nach der Angabe von Utz in o und Sa kai durch das Alkaliverfahren von Katheptase befreit wurde. Die Ergebnisse ergaben, dass die Peptonkörper auch durch diese von der Katheptase befreite Ereptase bei schwach alkalischer Reaktion deutlich abgespalten werden konnten (Tabelle 5). Die Lebermazeration, die durch das Säureverfahren von 


\section{Tabelle 5 .}

Wirkung der von Katheptase befreiten Ereptase auf Caseino-,

-. Mucino-, Fibrino- und Gelatopepton.

Die Lebermazeration des Schweins wurde nach dem Alkali-Verfahren bei $\mathbf{p H ~} \mathbf{9 , 0}$ (Uta $\mathrm{n}$ o und S a k a i, 19414) von Katheptase befreit, mit Aceton gefällt und getrocknet.

Die Peptonkörper ebenso wie in Tabelle 1 gebraucht.

Versuchsansatz: $20 \mathrm{cem} 1 \%$ iger Substratpufferlösung $(\mathrm{pH} \mathrm{7,4})+2 \mathrm{ccm}$ Ereptaselösung $(0,2 \mathrm{~g})+$ Toluol ; digeriert bei $37^{\circ} \mathrm{C}$.

Kongrollversuch: 2 ecm Ereptaselösung $(0,2 \mathrm{~g})$ mit Pufferlösung ohne Substrat auf $22 \mathrm{ccm}$ gebracht $(\mathrm{pH} 7,4)+$ Toluol; digeriert bei $37^{\circ} \mathrm{C}$. Kontrolle mit Enzym-Puffer ohrie Pepton. Formoltitration.

Die korrigierten Werte der Aciditätszunahme in Tabelle angegeben.

\begin{tabular}{|c|c|c|c|c|c|c|}
\hline \multicolumn{7}{|c|}{ Aciditätszunahme in $4 \mathrm{ccm}$ Digestat $(\mathrm{ccm} 0,1 \mathrm{n}-\mathrm{NajOH})$} \\
\hline Std. $_{\text {Substrate }}^{\text {Sute }}$ & $\begin{array}{c}\text { Caseino- } \\
\text { pepton } \\
\text { (Saft) }\end{array}$ & $\begin{array}{l}\text { Gelato- } \\
\text { pepton } \\
\text { (Brei) }\end{array}$ & $\begin{array}{l}\text { Mucino- } \\
\text { pepton } \\
\text { (Saft) }\end{array}$ & $\begin{array}{c}\text { Mucino- } \\
\text { pepton } \\
\text { (Brei) }\end{array}$ & $\begin{array}{l}\text { Fibrino- } \\
\text { pepton } \\
\text { (Saft) }\end{array}$ & $\begin{array}{l}\text { Fibrino- } \\
\text { pepton } \\
\text { (Brei) }\end{array}$ \\
\hline 24 & 0,4 & 0,2 & 0,9 & 0,3 & 0,4 & $-0,4$ \\
\hline 72 & 0,5 & 0,3 & 1,4 & 0,4 & 0,4 & 0,7 \\
\hline 120 & 0,8 & 0,4 & $\cdot 1,6$ & 0,5 & 0,6 & 0,9 \\
\hline
\end{tabular}

$$
\begin{gathered}
\text { Tabelle } 6 . \\
\text { Wirkung der von Ereptase befreiten Katheptase auf Caseino-, } \\
\text { Mucino-, Fibrino- und Gelatopepton. }
\end{gathered}
$$

Die Lebermazeration des Schweins wurde von ihrer Ereptase-Wirkung nach dem Säure-Verfahren bei pH 4,0 (Utzino und Saka i, 1941) ${ }^{4}$ ) befreit, mit Aceton gefällt und getrocknet.

Die Peptonkörper wie in Tabelle 1 gebraucht.

Versuchsansatz: $16 \mathrm{ccm} 2 \%$ iger Substratpufferlösung $(\mathrm{pH} 4,5)+2 \mathrm{ccm}$ Katheptaselösung $(0,2 \mathrm{~g})$, die mit $2 \mathrm{ccm} 0,1$ Mol-Cysteinlösung aktiviert wurde, oder $+2 \mathrm{~cm} \mathrm{Ka}$ theptaselösung mit $2 \mathrm{ccm}$ Wasser ohne Cystein + Toluol; digeriert bei $37^{\circ} \mathrm{C}$.

Kontrollversuch: 2 cem Katheptaselösung mit $2 \mathrm{ccm}$ 0, I Mol-Cysteinlösung oder

\begin{tabular}{|c|c|c|c|c|c|c|c|}
\hline \multicolumn{8}{|c|}{ Aciditatszunabme in $4 \mathrm{ccm}$ Digestat $(\mathrm{ccm} 0,1 \mathrm{n}-\mathrm{NaOH})$} \\
\hline Std. & & $\begin{array}{c}\text { Caseino- } \\
\text { pepton } \\
\text { (Saft) }\end{array}$ & $\begin{array}{l}\text { Gelato- } \\
\text { pepton } \\
\text { (Brei) }\end{array}$ & $\begin{array}{l}\text { Mucino- } \\
\text { pepton } \\
\text { (Satt) }\end{array}$ & $\begin{array}{c}\text { Mucino- } \\
\text { pepton } \\
\text { (Brei) }\end{array}$ & $\begin{array}{l}\text { Fibrino- } \\
\text { pepton } \\
\text { (Saft) }\end{array}$ & $\begin{array}{l}\text { Fibrino- } \\
\text { pepton } \\
\text { (Brei) }\end{array}$ \\
\hline $\begin{array}{l}\text { mit Cystein } \\
\text { aktiviert }\end{array}$ & $\begin{array}{r}24 \\
72 \\
120\end{array}$ & $\begin{array}{l}0,9 \\
1,3 \\
1,7\end{array}$ & $\begin{array}{l}0,4 \\
0,6 \\
0,8\end{array}$ & $\begin{array}{l}0,7 \\
0,9 \\
1,0\end{array}$ & $\begin{array}{l}0,2 \\
0,3 \\
0,4\end{array}$ & $\begin{array}{l}0,2 \\
0,2 \\
0,4\end{array}$ & $\begin{array}{l}1,1 \\
1,4 \\
1,8\end{array}$ \\
\hline $\begin{array}{c}\text { nicht aktiviert } \\
\text { (Kontrolle) }\end{array}$ & $\begin{array}{r}24 \\
72 \\
120\end{array}$ & $\begin{array}{l}0,3 \\
0,5 \\
0,8\end{array}$ & $\begin{array}{l}0,1 \\
0,2 \\
0,3\end{array}$ & $\begin{array}{l}0,9 \\
0,4 \\
0,5\end{array}$ & $\begin{array}{l}0,1 \\
0,2 \\
0,3\end{array}$ & $\begin{array}{l}0,1 \\
0,2 \\
0,2\end{array}$ & $\begin{array}{l}0,2 \\
0,3 \\
0,5\end{array}$ \\
\hline
\end{tabular}
nur mit $2 \mathrm{ccm}$ Wasser ohne Cystein $+16 \mathrm{~cm}$ Pufferlösung ohne Substrat+Toluol; digeriert bei $37^{\circ} \mathrm{C}$. 
Ereptase getrennt wurde, griff auch sehr stark diese Peptonkörper ein und diese Pepton-Hydrolyse wurde durch Zusatz von Cystein deutlich befördert (Tabelle 6).

Auf Grund dieser Ergebnisse kann man sehr wahrscheinlich die Cysteinaktivierung der Peptonhydrolyse bei schwach saurer Reaktion annehmen und eine Kathopeptonase (katheptische Peptonase)im Sinne von Utzin o und Sakai (1941) $\left.{ }^{4}\right)$ bestätigen.

Falls man den Einfluss der Darstellungsweise des Peptons auf die Enzymwirkung vergleichend in Rücksicht zieht, wurde einmal das durch den Magensaft bewirkte Pepton wie Caseino- und Mucino-Pepton durch den Darmsaft stärker hydrolysiert als das durch Schleimhautmazeration des Magens abgebaute, das mittels der Mazeration dargestellté Fibrino-Pepton aber dadurch stärker als das Saft-Pepton angegriffen. DasselbeResultat wurde auch bei den Versuchen mit Gewebespeptonase erzielt. Die Frage über die Ursache dieses Unterschieds bleibt aber noch ungeklärt. Bei der vergleichenden Beobachtung scheinen die stärkste Hydrolyse des Caseino-Peptons (Saft), Mncino-Peptons (Saft) und auch des Fibrino-Peptons (Brei) fast im gleichen Grad sich zu vollziehen, während bei der Gewebesereptase-Wirkung nur die des MucinoPeptons(Saft)im demselben Grad auftrat, die des anderen Peptons aber deutlich schwächer, also fast in der Hälfte der stärksten Hydrolyse des Mucino-Peptons. Bei der Kathopeptonase-Wirkung war die Spaltung des Caseino-Peptons (Saft) und des Fibrino-Peptons(Brei) am stärksten und fast im gleichen Grad wie die bei der Darmsaftwirkung, während die des Mucino-Peptons (Brei) und des Fibrino-Peptons (Saft) trotz der Cysteinaktivierung sehr niedrig standen. Hier sei aber erwähnt, dass diese Gewebespeptonase nach der Säure- oder Alkali-Behandlung zur Anwendung kam und die Beeinträchtigung ihrer Aktivität nicht sicher ausgeschlossen werden konnte. In guter Übereinstimmung mitden oben angeführten Ergebnissen wurde das Verhalten des Mucino-Peptons (Saft) gegenüber diesen Enzymwirkungen und auch dem Nierenenzym bestätigt, was besonders in Tabelle 7 zusammengefasst ist.

\section{Tabelle 7. \\ Das Verhalten des Mucinopeptons (Saft) gegenüber verschiedenen Enzymarten.}

Das Mucin, welches aus Magenschleimhautextrakt dargestellt wurde, hat man der maximalen Digestion mit Hundemagensaft unterworfen und das Spaltprodukt mit Alkohol gefällt und getrocknet. Die Gewebe-Mazeration besteht aus 1 Teil Gewebesbrei und 3 Volumteilen Glycerin-Wasser $(1: 1)$. Die Versuche bei pH 4,5 wurden hier ohne Cysteinzusatz ausgeführt. Der Pankreassaft wurde aus der Pankreasfistel und 
der Darmsaft aus der Thiry - Vella'schen Darmschlinge des Hundes gewonnen. Der erstere wurde mit Enterokinase aktiviert.

Versuchsansatz: $20 \mathrm{ccm} 1 \%$ iger Substratpufferlösung $+2 \mathrm{ccm}$ Mazeration oder Saft + Tolnol; digeriert bei $37^{\circ} \mathrm{C}$.

Kontrolle mit Enzym:Puffer ohne Substrat. Formoltitration.

\begin{tabular}{|c|c|c|c|c|c|c|c|c|c|c|}
\hline \multicolumn{11}{|c|}{ Aciditätszunahme in $4 \mathrm{ecm}$ Digestat (cm $0,1 \mathrm{n}-\mathrm{NaOH}$ ) } \\
\hline \multirow[b]{2}{*}{ Std. } & \multicolumn{2}{|c|}{$\begin{array}{l}\text { Magenschleim- } \\
\text { hautmazeration } \\
\text { (Hund) } \begin{array}{c}\text { (Kanin- } \\
\text { chen) }\end{array}\end{array}$} & \multirow{2}{*}{$\frac{\begin{array}{c}\text { Darm- } \\
\text { saft } \\
\text { (Hund) }\end{array}}{\left(\begin{array}{c}\text { Hund } \\
7,5\end{array}\right.}$} & \multirow{2}{*}{$\begin{array}{c}\text { Pankreas- } \\
\text { saft }+ \\
\text { Kinase } \\
\text { (Hund) } \\
7,5\end{array}$} & \multicolumn{2}{|c|}{$\begin{array}{l}\text { Pankreas: } \\
\text { mazeration } \\
\text { (Schwein) }\end{array}$} & \multicolumn{2}{|c|}{$\begin{array}{c}\text { Leber- } \\
\text { mazeration } \\
\text { (Schwein) }\end{array}$} & \multicolumn{2}{|c|}{\begin{tabular}{|l} 
Nieren \\
mazeration \\
(Sehwein)
\end{tabular}} \\
\hline & 2 & 2 & & & 4,5 & 7,5 & 4,5 & 7,5 & 4,5 & 7,5 \\
\hline 24 & 0 & 0 & 0,4 & 0,3 & 0,2 & 0,5 & 0,5 & 0,6 & 0,4 & 0,7 \\
\hline 72 & 0 & 0 & 0,5 & 0,3 & 0,2 & 0,7 & 0,5 . & 0,8 & 0,4 & 0,8 \\
\hline 120 & 0 & 0 & 0,6 & 0,4 & 0,3 & 0,7 & 0,6 & 0,9 & 0,5 & 0,8 \\
\hline
\end{tabular}

\section{Zusammenfassung.}

1. In vorliegender Arbeit wurde das enzymologische Verhalten derPeptonkörper, die nach wiederholter Einwirkung des Hundemagensaftes hergestellt wurden, gegenüber den Proteasenwirkungen verschiedener Herkunft studiert.

Dem Magensaft wie der Mazeration der Magenschleimhaut widerstehen die Peptonkörper, während sie durch den Pankreas- wie durch den Darmsaft des Hundes deutlich angegriffen werden, wobei die Aciditätszunahme durch den Darmsaft viel stärker als die durch den Pankreassaft war.

2. Hier sei besonders hervorgehoben, dass diese Peptonkörper leicht durch die von der Katheptase befreite Ereptase des Gewebes bei $\mathrm{pH} \mathrm{7,5}$ und auch durch die von der Ereptase befreite Katheptase bei $\mathrm{pH}$ 4,5 angegriffen werden. Diese Beobachtung lässt wohl an die Angabe von Utzino und Sakai (1941) $)^{4}$ erinnern, dass sich in Geweben eine katheptische Peptonase (kurz Kathopeptonase) befindet.

\section{Literaturr.}

(1) Itzioka, F., Journ. Biochem., 1937, 25, 329.

(2) Mori, T., ibid., 1938, 28, 205.

(3) Che n, T.-T., Hirook a, T. u. K a w ah ar ada, M., ibid., 1940, 31, 335.

(4) Utzino, S. u. Sakai, T., ibid., 1941, 33, 457.

(5) Waldsehmidt-Leitz, E. u. Simons, E., Ztschr. Physiol. Chem., 1926, 156, 99. 
(6) Waldschmidt-Leitz, E. u. Waldschmidt-Graser, J., ibid., 1927, 166, 241.

(7) Waldsehmidt-Leitz, E. u. Harteneek, A., Ztschr. Physiol. Chem., 1925, 149, 203; Waldschmidt-Leitz, E. u. Sch äf f n er, A., ibid., 1926, 151, 31.

(8) Matsuo, M., Journ. Biochem., 1941, 33, 59.

(9) Waldschmidt-Leitz, E., Sehäfner, A., Beck,J.J.u. Blum, E., Zeits. Physiol. Chem., 1929, 188, 17. 